\title{
HISTORIA Y CIUDADANÍA EN EL BACHILLERATO MEXICANO. UNA MIRADA DESDE LA PEDAGOGÍA POR LA JUSTICIA SOCIAL
}

\author{
HISTORY AND CITIZENSHIP IN MEXICAN HIGH SCHOOL. \\ A VIEW FROM SOCIAL JUSTICE PEDAGOGY
}

\begin{abstract}
Sebastián Plá*
El presente texto es un análisis curricular de la asignatura de historia y su relación con la formación ciudadana en el Bachillerato General mexicano. Para el análisis se considera el currículo en su dimensión histórica y política. Se trabaja en específico un contenido histórico, la colonialidad, y se pregunta si la forma en que se prescribe su enseñanza es potencialmente promotora o no de la justicia social. La conclusión es que la racionalidad de la enseñanza de la historia promueve una estrategia adaptativa que obstaculiza la comprensión de las relaciones de poder en la sociedad mexicana actual y por tanto no da elementos para la transformación social, mientras que la formación ciudadana sí problematiza la historia colonial y su impacto en el México contemporáneo, pero la racionalidad que subyace en la idea de ciudadano, no necesariamente promueve acciones transformativas de fondo. El análisis se realiza a partir de una metodología cualitativa basada en la pedagogía por la justicia social.
\end{abstract}

Palabras claves: Enseñanza de la historia, formación ciudadana, Bachillerato General, México, justicia social.

This text is a curricular analysis of the course of history and its relationship with citizenship education in the Mexican High School. For the analysis considers the curriculum in its historical and political dimension to describe how the two subjects have two antinomic rationalities. Coloniality is analyzed within syllabus and asks whether the way teaching is prescribed is potentially promoter or social justice. The conclusion is that the rationality of history education promotes an adaptive strategy that hinders the understanding of power relations in contemporary Mexican society and therefore does not provide elements for social change. By contrast citizenship education problematizes colonial history and its impact on contemporary Mexico, but the rationality that underlies the idea of citizen, not necessarily promote transformative actions background. This is because the Mexican school resigned from the 2008 reform, the whole critical tradition of social studies. This study is supported from a qualitative methodology based on social justice pedagogy.

Key words: Teaching History, Citinzenship Education, High School, Mexico, Social Justice.

Las relaciones entre enseñanza de la historia y formación ciudadana han sido, en México y en otras muchas partes del mundo, muy estrechas, pero en ningún momento fijas. Su proximidad educativa se debe, más allá de si se organizan en una misma asignatura o en diferentes programas de estudio, en que ambas tienen el mismo fin: formar ciudadanos. En el ámbito del diseño curricular, las relaciones entre ambas han variado con el tiempo. En una muy apretada síntesis del caso mexicano, las necesidades de crear una identidad nacional en el último tercio del siglo XIX, tras dos intervenciones extranjeras, la pérdida de mitad del territorio y décadas de luchas intestinas que arrojaron como vencedores a los liberales, sometieron a la historia escolar a la función básica de la creación del panteón patrio. Este principio fundacional perdura, aunque ya no ocupa el mismo lugar jerárquico que entonces. Se modificó en la posrevolución (1920-1940) y regresó con ahínco en el proyecto de Unidad Nacional (1940-1970), cuando se sedimentaron en la escuela la teleología mestiza y los estereotipos que hoy nos identifican. Ahí la historia y el civismo iban de la mano, a pesar de ser dos asignaturas distintas. Los años setenta juntaron las asignaturas en primaria y secundaria, pero las mantuvieron separadas en bachillerato. No fue hasta fines del siglo XX, con las reformas educativas de gran calado que ha sufrido México en los últimos veinticinco años, que la historia y la formación ciudadana se separaron en cuanto sus formas de pensar la identidad y la ciudadanía. La primera retuvo su código disciplinar ${ }^{1}$, pero con modificaciones importantes, sobre todo en lo

* Universidad Nacional Autónoma de México (UNAM). Instituto de Investigaciones sobre la Universidad y la Educación (IISUE). Ciudad de México, México. Correo electrónico: sebastianpla@gmail.com 
cognitivo, mientras que la segunda fue sustituida por la enseñanza de ética y de valores, especialmente con la reforma a la asignatura de 1999 en el nivel de secundaria.

El presente texto estudia esta separación. Para eso, indaga acerca de cómo se imbrican las tradiciones de dichas asignaturas en los programas de estudio del Bachillerato General en México y las implicaciones que tiene para la pedagogía crítica. He organizado el artículo en cinco apartados: en los dos primeros defino la mirada pedagógica y las herramientas teóricas que utilizo, en especial la pedagogía por la justicia social. Posteriormente hago un recuento general de tres grandes tradiciones de enseñanza de la historia y de la formación ciudadana, para que a partir de ellas puedan entenderse las estrategias o finalidades adaptativas, afirmativas y transformativas que presentan los programas de estudio. En seguida, describo brevemente al bachillerato general y el enfoque de competencias que entró en vigor en 2008. Por último, analizo un solo contenido que se imparte tanto en la asignatura de formación ciudadana y valoral como en las de Historia Universal y de México: el colonialismo. Como se podrá observar, el resultado curricular de la enseñanza de la historia responde más al largo pasado de tradiciones docentes y de una función social de la historia meramente identitaria, por lo que no promueve una reflexión crítica en los estudiantes. En cambio, las visiones ciudadanas de las asignaturas filosóficas han logrado rompen con la tradición histórica de la educación cívica. El resultado es una enseñanza de la historia que continúa reproduciendo lógicas excluyentes y convirtiéndose, una vez más, en un obstáculo para modificar a largo plazo las relaciones simbólicas entre diversas identidades en el México contemporáneo.

\section{Una mirada desde la pedagogía por la justicia social}

La pedagogía por la justicia social es una propuesta abierta que retoma diversos enfoques teóricos. No es una propuesta uniforme ni cerrada a un solo enfoque teórico, como el posestructuralismo, los estudios culturales, el poscolonialismo, la decolonialidad o la pedagogía crítica. Independientemente de esta pluralidad, todos comparten la idea de luchar contra las formas de dominación en el mundo contemporáneo. De este criterio unificador se desprenden cuatro finalidades básicas de la pedagogía por la justicia social: a) desafiar, confrontar y producir rupturas en los estereotipos, las falsedades y los conceptos erróneos que guían las desigualdades y las discriminaciones estructurales basadas en clase social, género, fenotipo, cultura y otras diferencias entre los seres humanos; b) proveer los elementos educativos necesarios para que todos los estudiantes puedan desarrollar su potencial; c) aprovechar las fortalezas de los estudiantes en su propia educación; y d) crear ambientes de aprendizaje que promuevan el pensamiento crítico como agente del cambio social (Chapman and Hobbel, 2010:1-2). Sobre el primer punto trata este trabajo.

La relación entre el currículum y la sociedad a la que pertenece se puede observar desde una pedagogía de la justicia social, si se analiza el tipo de estrategia de cambio social que promueve. Nancy Fraser sostiene que básicamente hay dos: la afirmativa y la transformativa:

las estrategias afirmativas para reparar la injusticia intentan corregir los resultados desiguales de los acuerdos sociales sin tocar las estructuras sociales subyacentes que los generan. En cambio, las estrategias transformadoras aspiran a corregir los resultados injustos reestructurando, precisamente, el marco generador subyacente (Fraser 2006: 72).

Mientras las primeras se centran en el resultado, las segundas buscan la transformación de las causas últimas que producen la desigualdad. Sin embargo, la historia del desarrollismo latinoamericano de mediados del siglo XX creó una tercera estrategia: la adaptativa. Esta puede ser entendida como aquella donde la movilidad social está paralizada por un orden simbólico y de producción preestablecido, pero en el que un Estado, por lo general autoritario, puede crear condiciones de relativa redistribución de la riqueza, como el caso mexicano. Al analizar las relaciones entre formación valoral y enseñanza de la historia en el Bachillerato General (BG) mexicano, podremos observar que estas tres estrategias se imbrican en un mismo programa de estudio, aunque en última instancia predomina la afirmativa, más acorde a una idea de ciudadanía basada en un conjunto de habilidades cognitivas, que permite al estudiante desenvolverse en la sociedad desigual del presente. 


\section{Nota teórica metodológica}

Los estudios del currículum, desde su creación en Estados Unidos de América en las primeras dos décadas del siglo XX, dividió sus referentes empíricos en dos grandes ramas. Por un lado, los estudios centrados en la experiencia escolar y desarrollados en un inicio por Johan Dewey. Por otro lado, se desarrollaron los estudios de la organización escolar y en especial de los programas de estudio, a partir de los trabajos de Franklin Bobbit (Díaz Barriga 2015). Ambas ramas han tenido, a lo largo del siglo XX, importantes renovaciones teóricas y metodológicas. Acerca de la segunda dimensión, los estudios respecto de la organización y planeación educativas, que es donde se enmarca mi artículo, quizá la corriente de mayor influencia ha sido el análisis sistemático y técnico propuesto por Ralph Tyler y seguidores. En oposición a esta corriente, los años setenta vieron producirse una ruptura en los estudios del currículum, con quienes se denominaron en un inicio los reconceptualizadores ${ }^{2}$. El principal objetivo de este grupo fue romper con la visión técnica del control escolar y analizar el currículum como construcción política, histórica y cultural. La variedad de estudiosos ubicados aquí es muy amplia, pero todos tienen en común cuatro características.

La primera es que todos los autores, reconociendo las relaciones de poder dentro de un currículum, rechazan la visión monolítica del mismo, en el que un grupo autorregulado produce de manera armónica una propuesta curricular. Por el contrario, el resultado programático es un momento coyuntural que mitiga los antagonismos bajo principios hegemónicos que los articulan. Este proceso lo vemos a aquí a partir de la noción de ciudadanía y la enseñanza de la historia que se prescribe para el BG mexicano. Un segundo aspecto es el reconocimiento de múltiples lecturas posibles del currículum que desbordan por mucho las limitaciones de las teorías de la reproducción. Entre estas lecturas pueden encontrarse visiones históricas, políticas, de género, fenomenológicas (García Garduño, 2014) y, en los últimos tiempos, desde la justicia social. En el presente trabajo me limito a la dimensión histórica y política del currículum.

Una tercera característica de este grupo es que las metodologías utilizadas para el análisis también son diversas y basadas en procedencias teóricas varias, aunque predomina el estudio cualitativo y de enorme carga teórica. De esta manera, más que usar una teoría particular o un método preestablecido, se construyen herramientas teóricas que permiten establecer categorías analíticas intermedias para vincular referentes empíricos, preguntas de investigación y referentes teóricos. En mi caso se retoman las estrategias de la filosofía política de Fraser, para mirar la potencialidad de justicia curricular de dos asignaturas. Por último, se rompe con el esencialismo positivista de la neutralidad científica y reconocen explícitamente su compromiso político. Incluso, consideran este aspecto como parte irreductible de su epistemología. Esta posición es, en la inmensa mayoría de los autores citados, un compromiso con los sectores marginados, dominados, explotados o excluidos por la sociedad capitalista actual.

Basado en estas cuatro características he diseñado una matriz de análisis para estudiar las interpretaciones de las narraciones históricas curriculares, desde una mirada de la pedagogía por la justicia social. El objetivo es observar la potencialidad de un diseño curricular para reparar "la subordinación de estatus mediante la deconstrucción de las oposiciones simbólicas que subyacen a los patrones de valor institucionalizados en la actualidad" (Fraser 2006: 73). Para esto se lee el currículum desde las dimensiones políticas e históricas de la enseñanza de la historia y la formación cívica y valoral, consideradas, en este caso, a partir de las tres estrategias de justicia social -adaptativa, afirmativa y transformativa- que promueven. La dimensión histórica permite estudiar las condiciones de posibilidad para la producción de las antinomias. La dimensión política identifica el antagonismo político, lo que termina por generar en el hecho educativo tensiones irresolubles solo mitigadas por la coyuntura específica.

Por la cantidad de información que arroja el enfoque de investigación cualitativo y la lectura del currículum basado en las dimensiones histórica y política, fue necesario delimitar el estudio al Bachillerato General mexicano. Asimismo, se seleccionó un contenido histórico -colonialismo y colonialidad-, por dos razones: a) es uno de los pocos contenidos explícitos con contenidos en los programas de Ética y Valores ${ }^{3}$, b) el significado histórico de la conquista, el colonialismo y la colonialidad para el presente, y en especial para la institucionalización de la subordinación de estatus en el México actual. Por tanto, la selección de este contenido permite la evaluación de su potencial adaptativo, afirmativo o transformativo. 


\section{Adaptación, afirmación y transformación ${ }^{4}$}

Las tres grandes agrupaciones de enseñanza de la historia que explico a continuación tienen una correlación directa con ideales distintos de ciudadanía y de estrategia por la justicia social. $\mathrm{Al}$ primer grupo, el más poderoso por su estrecho vínculo con las instituciones del Estado-nación, lo denomino adaptativo (historia nacional) y tiene su origen en México en la segunda mitad del siglo XIX. El segundo, basado en la ciencia, es el grupo afirmativo, cuyo concepto de ciudadanía proviene del liberalismo, pero su propuesta de enseñanza de la historia emerge en los años noventa del siglo XX. El tercer grupo es la propuesta transformativa o crítica, que se desarrolla en México con el movimiento posrevolucionario de los años treinta hasta mediados del siglo $\mathrm{XX}$, pero que hoy se encuentra fuera de buena parte del sistema educativo. Naturalmente, esta división arbitraria tiene numerosos umbrales donde las fronteras se diluyen. Sin embargo, el núcleo de cada una es independiente de la otra.

\section{Adaptación}

La postura nacionalista de la enseñanza de la historia y la formación cívica es la más añeja y perdurable. En México, su función social primordial ha sido la construcción de la identidad nacional creando un panteón patrio y una secuencia narrativa teleológica. Su construcción no necesariamente fue pacífica y quizá el hecho histórico más determinante fue el triunfo liberal de 1867 (O'Gorman 1986). Con la Ley Orgánica de la Instrucción Pública en el Distrito Federal de 1867, se da inicio a la historia y la educación moral. En ella se establecieron aspectos ciudadanos relevantes: laicismo, republicanismo, democracia -más en el papel que en la práctica-y la adoración a los símbolos patrios. Asimismo se instauró la dicotomía nacional/extranjero con la separación de dos asignaturas: Historia de México e Historia Universal (Sánchez Quintanar 2004), situación que se repitió en la recién fundada Escuela Nacional Preparatoria (ENP). Sin embargo, la historia nacional seguía en disputa. Tras décadas de enfrentamientos políticos e historiográficos acerca del origen de lo mexicano: lo prehispánico, lo español o la combinación mestiza, el proyecto de Unidad Nacional (1940-1970) escogió la última (Vásquez 1970).
En la homogeneidad impuesta por la historia nacional, la concepción ciudadana se reduce a un civismo formal de cumplimiento de las obligaciones y acceso de los derechos otorgados por la Constitución política. En este tipo de historia y ciudadanía escolar, la historiografía profesional ha cumplido un papel muy relevante a lo largo de la historia mexicana. Por ejemplo, Patricia Galeana, directora del Instituto Nacional de Estudios Históricos de las Revoluciones Mexicanas (INHERM), se lamentaba al inicio de su gestión que "todos los aspectos de grandeza se omitieron por hacer una historia supuestamente desacralizadora y acabar con la historia de bronce", por lo que los mexicanos quedaron en orfandad (Miranda 2013). Los contenidos de esta historia son los relacionados con el Estado-nación y las habilidades cognitivas no son explícitas, aunque desde hace tres décadas se tiende a imbricar método histórico, historia patria y corriente pedagógica en boga. La estrategia de justicia social predominante es la adaptativa, donde el ciudadano garantiza la preservación del Estado y este vela a su vez por él. La postura nacionalista carece por completo de investigación para sustentar sus supuestos de que a mayor identidad nacional mayor justicia social.

\section{Afirmación}

En sentido opuesto, el posicionamiento científico, tanto a nivel nacional como internacional, es el que más sustento tiene en investigaciones empíricas, por lo menos en lo referente a los aprendizajes. Parte importante de este logro se debe a que en este grupo de investigación se incluyen estudios con orígenes disciplinares muy diversos y que el propio concepto de ciudadanía, centrado en el sujeto liberal, encuentra múltiples interpretaciones y posibilidades pedagógicas. Por ejemplo, en el ámbito anglosajón podemos incluir aquí los posicionamientos de Peter Seixas (2013), Stéphane Levesque (2008) y Keith Barton y Linda Levstik (2004), quienes conciben que la finalidad de la educación es dar al estudiante las competencias y habilidades necesarias para poder enfrentar las condiciones de la sociedad contemporánea. Unos lo harán más desde las habilidades cognitivas tomadas de la disciplina histórica; otros combinarán habilidades cognitivas y ética ciudadana; y los últimos lo harán más preocupados por la agencia de los individuos en una ciudadanía participativa. En México, esta corriente empezó en los años noventa y la investigadora más relevante es, 
desde el constructivismo, Frida Díaz Barriga (1998, 2008). En relación con la formación ciudadana, la tradición mexicana es muy larga, pero una autora relevante e influyente hoy es Silvia Conde (2015), aunque el impacto de la formación valoral para una sociedad racional de autores como Bonifacio Barba, son significativas (2007). Este cambio de formación cívica a formación valoral será importante en las futuras imbricaciones entre historia y ciudadanía en la escuela.

A riesgo de simplificar, podríamos decir que para este grupo de investigadores la finalidad de la enseñanza de la historia es ofrecer las herramientas que requiere el pensamiento crítico para poder participar activamente en la sociedad. El objetivo cardinal es el desarrollo de habilidades de pensamiento y de competencias ciudadanas. Las competencias se congregan en la corriente pragmática de pensamiento crítico, es decir, el fomento del aprendizaje cognitivo en donde se alcanza un pensamiento racional, basado en criterios objetivos de verdad (Boisvert 2004). Esto permite vincular la historia y sus criterios metodológicos de verdad, con la formación para una ciudadanía objetiva. El concepto de ciudadanía implica una identidad sociopolítica en la que el Estado existe para garantizar los derechos de sus ciudadanos y es responsabilidad individual cumplir con las obligaciones y hacer valer sus derechos. El contenido histórico, aunque no se niega su importancia, es subsidiario del proceso de pensamiento. Las estrategias que promueven son de carácter afirmativa, pues la justicia social se da dentro de una democracia y un mercado liberal competitivo, en el que el desempeño de cada uno determina la posición que ocupa en la sociedad.

\section{Transformación}

Por el contrario, la enseñanza crítica o transformativa centrará su propuesta en los derechos sociales, sin menoscabar los derechos políticos ni civiles. No se preocupan tanto por el individuo como por el conjunto de la sociedad y de las relaciones de poder que producen la desigualdad y la injusticia social. Al igual que el grupo ubicado bajo el título de científicos liberales, los posicionamientos teóricos y metodológicos del grupo crítico son muy diversos, aunque muchos encuentran en los escritos de Paulo Freire una fuente de inspiración significativa. Por lo general, sus posicionamientos se inclinan más hacia los estudios sociales, donde ubican a la historia, que a las didácticas disciplinares específicas. Internacionalmente hay demasiados autores y caminos divergentes, pero algunos relevantes son en estudios sociales de Wayne Ross (2001, DeLeon y Ross 2010), en enseñanza de la historia de Terrie Epstein (2009), en formación ciudadana de Vanessa Andreotti (2012) o las teorías decoloniales alrededor de los trabajos de Catherine Walsh (2009). Como programas de estudio, quizá las reformas educativas de Ecuador y Bolivia sean ejemplo de lo anterior (Plá, 2016). En México, fuera de los estudios acerca de educación indígena, hay muy poco trabajado respecto de la enseñanza de la historia y la formación ciudadana desde una perspectiva crítica. Sin embargo, la historia de México no ha quedado exenta de este posicionamiento político y educativo. La educación socialista (19341940), sobre todo durante el gobierno de Lázaro Cárdenas, dio impulso al conocimiento histórico y social para la búsqueda de la justicia social, el pensamiento educativo de Rafael Ramírez (1948) y la influencia del materialismo histórico también fueron importantes. En los años setenta se realizó un cambio curricular donde se organizó el conocimiento por áreas y dio entrada a la escuela activa, lo que produjo un fenómeno paradójico: autoritarismo político y contenido crítico en la escuela.

La formación para la ciudadanía crítica da mucho peso a las colectividades, a la concientización concerniente a las relaciones de poder y las injusticias que produce. Se puede sostener que la formación para la ciudadanía crítica afirma que si no hay una justa redistribución de la riqueza a partir de los derechos sociales (educación, vivienda, salud, trabajo) y no hay modificaciones de fondo al sistema capitalista, no existen las condiciones para la justicia social. Debido a que la escuela no cambiará la base económica, el objetivo de la formación ciudadana crítica es enseñar a identificar las condiciones materiales que impiden la igualdad, para así deconstruir las relaciones simbólicas que legitiman las estructuras sociales de producción y de reproducción de la desigualdad. Para ellos, los contenidos históricos son centrales, pues son las interpretaciones del pasado y del presente político, social, económico y cultural, la materia prima para la deconstrucción de los opuestos binarios que legitiman las relaciones de poder. El colonialismo, el orden patriarcal o la mala distribución de la riqueza son temas de los que hay que aprender y pensar desde diversas posiciones epistemológicas como el decolonialismo, 
la deconstrucción o la dialéctica, entre otros. No hay universales cognitivos ni valorales.

El resumen de estas tres posiciones atinentes a la relación entre enseñanza de la historia y formación ciudadana, aunque esquemática y por tanto porosa, me permitirá analizar un plan de estudio y varios programas de asignaturas de un subsistema de EMS superior, para demostrar que las visiones, en ocasiones simplistas de un isomorfismo universal de las reformas educativas, se desmoronan ante análisis históricos y genealógicos de la historia del currículo. Asimismo, el análisis del currículo me permitirá ver si los contenidos históricos pueden o no ser considerados como potencialmente más favorecedores de la justicia social.

\section{La RIEMS y el BG}

No es tema de este artículo la historia del bachillerato mexicano, sino los programas de historia en el presente. Sin embargo, es necesario tener en cuenta algunos rasgos centrales del pasado para poder comprender las transformaciones en el diseño curricular de las asignaturas de Historia y Educación y Valores en este nivel educativo, y así poder observar por qué la historia conserva su código disciplinar y continúa reproduciendo estrategias adaptativas, a diferencia de la formación ética y ciudadana.

El bachillerato mexicano, a diferencia del pasado relativamente homogéneo de la educación básica, tiene una historia desperdigada. Asimismo, encuentra su eclosión en tiempos recientes, los años setenta del siglo XX. Si no consideramos la primera mitad del siglo XIX, cuando no había un sistema educativo propiamente dicho, podemos seguir la propuesta de Lorenza Villa Lever (2010) y sus etapas de la historia del bachillerato mexicano. La primera etapa (1867-1878) se centra en la Escuela Nacional Preparatoria (ENP), fundada en 1867 bajo el positivismo de Gabino Barreda y el gobierno liberal de Benito Juárez. En ella, aunque relegada por las ciencias, la historia ocupó un lugar en el currículo que no ha abandonado hasta la fecha (Meneses 1998: 236). Durante el segundo periodo (1878-1948), el nivel se divide en dos: la educación secundaria, destinada a la formación de ciudadanos en un sentido amplio y la preparatoria, de corte enciclopédico y propedéutico, donde la historia se asentó y cumplió su función de formadora del ciudadano nacional. En este mismo periodo, surgen los bachilleratos tecnológicos, dividiendo la identidad del nivel educativo entre una destinada al trabajo manual y otra de corte elitista, con un enfoque propedéutico.

La tercera etapa (1948-1989) es, sin lugar a dudas, la más relevante para la Educación Media Superior, como se comienza a llamar al bachillerato mexicano desde esta época (Villa Lever 2010). En ella, empujada por el aumento acelerado de la demanda, el nivel educativo comienza su masificación. En respuesta, la SEP crea diversas modalidades del subsistema: aumenta la educación tecnológica; crea un bachillerato terminal con enfoque profesional, el Colegio Nacional de Educación Técnica Profesional (Conalep); funda el Colegio de Bachilleres con la finalidad de ofrecer un Bachillerato General terminal y mantiene y amplía su oferta propedéutica en todo el país ${ }^{5}$. Uno de los problemas de esta diversificación fue la incapacidad del gobierno federal por dar una identidad propia al nivel educativo. Otra, relevante para este estudio, es que las asignaturas de historia, tanto en los bachilleratos universitarios de reciente creación como en el Colegio de Bachilleres, tuvieron una marcada impronta del pensamiento crítico, entendido en su dimensión política (Argüello 2017). Esto no eliminó la impronta adaptativa de la historia en muchas otras modalidades. La cuarta etapa (1989 a la actualidad $)^{6}$ mantiene el crecimiento acelerado del sistema, pero hay un esfuerzo deliberado por dar identidad al sistema: se crea la Subsecretaría de Educación Media Superior y, especialmente, se introduce el enfoque por competencias al subsistema. El primer esfuerzo lo vive el Conalep en 1993, pero no es con la Reforma Integral a la Educación Media Superior (RIEMS), la creación del Sistema Nacional de Bachillerato (SNB) y en especial el Marco Curricular Común (MCC), cuando las competencias se tornaron hegemónicas. En este periodo, la lógica adaptativa de las políticas educativas, desplaza a la paradoja autoritarismo/pensamiento crítico de los años setenta ${ }^{7}$.

La RIEMS y el MCC -y su enfoque por competencias- son un esfuerzo explícito por vincular de manera más explícita este nivel educativo con el mercado laboral global que exige la sociedad del conocimiento (SEP 2008a), respetando la historia desperdigada de la EMS. De esta manera, se respetaba la cultura escolar de cada bachillerato, siempre y cuando diseñaran sus planes y programas de estudio a partir de las competencias -genéricas, disciplinares y profesionales- y de cuatro campos disciplinares 
-Matemáticas, Ciencias experimentales, Ciencias sociales y Comunicación-, aunque posteriormente ante la presión social, especialmente por parte de los filósofos, se agregó un quinto -Humanidades(Pérez Rocha 2016).

Las competencias establecidas por la RIEMS, es decir, "la integración de habilidades, conocimientos y actitudes en un contexto específico" (SEP 2008a: 1) permiten definir estándares de desempeño que, independientemente de cada modelo educativo que se curse, los alumnos deben alcanzar. Las competencias genéricas son una especie de todo: son claves porque sirven para cualquier ámbito académico o laboral, son transversales a todas las disciplinas y son transferibles, pues ayudan a adquirir nuevas competencias. Desde esta lógica, son un estado cognitivo superior universal que facilita el desarrollo autónomo de los individuos. Sin embargo, en la práctica educativa del bachillerato general, la universalidad se desmembraba ante la historia propedéutica de los programas de estudio, por lo que se crearon competencias disciplinares básicas y extendidas, las primeras son las que deben aprender todos los estudiantes que cursan la EMS y las segundas son las que responden a los enfoques y perfiles de los distintos subsistemas (SEP 2008b). De nuevo las particularidades del bachillerato mexicano saltan a la vista: las competencias disciplinares o laborales, que son formas de conocer, hacer y ser con base en el desarrollo histórico de determinadas profesiones, permiten la invención didáctica de nuevas competencias de acuerdo con la cultura escolar de cada subsistema.

Basado en lo anterior, y para analizar las visiones respecto de la enseñanza de la historia y la formación ciudadana que se tejen en el BG mexicano desde la perspectiva de la justicia social en el currículo, debemos tener presente tres aspectos centrales de los programas de estudio. Primero, que incluyen las exigencias de MCC, que a su vez siguen procesos globales de reforma educativa. Segundo, que el resultado es algo nuevo que no debe pensarse como una simple continuidad de elementos o en su caso como la superación de tradiciones, sino que hay un reordenamiento de las diferentes componentes para construir nuevos significados. En tercer lugar, hay que tener presente que todo enfoque de competencias es por principio una estrategia afirmativa, pues su función es dar las habilidades necesarias para poder competir en igualdad de condiciones en un sistema económico preestablecido.

\section{Contenidos}

Los temas históricos y su relación con la formación ciudadana en los programas analizados responden a tradiciones diversas, por lo que pueden verse aspectos de la postura nacionalista-adaptativa, otros de la científica-afirmativa y de la casi extinta, crítica -transformativa-. Asimismo, si tenemos en cuenta las inconsistencias internas y externas de los diseños curriculares, la dificultad analítica aparece. Quizá la inconsistencia interna más notable esté en la relación entre la fundamentación de los programas, donde se expone la importancia de la formación ciudadana y la casi omisión total de estos contenidos en los apartados temáticos. Es decir, podría afirmarse que el gran defecto de estos programas es la ausencia de formación ciudadana, aunque esto es relativo por dos aspectos: a) se trabajan someramente en las sugerencias didácticas (BG 2013c), b) los silencios son síntoma de un cambio histórico en el que la formación ciudadana pasó de centrarse en el conocimiento de lo social, a un conjunto de habilidades que dan agencia para la participación cívica. Sin embargo, esta última afirmación también tiene matices, como muestro a continuación a partir de un ejemplo concreto: la colonialidad.

La colonialidad deriva del colonialismo, es decir, del proceso de expansión europea iniciado en la segunda mitad del siglo XV. En el caso mexicano, los trescientos años de dominación española durante el periodo colonial establecieron ciertos principios simbólicos que jerarquizaron las relaciones sociales y la distribución de la riqueza con base en la raza y el tipo de saber occidental, que ya entrado el siglo XVIII fue el iluminismo. La colonialidad hace referencia al reordenamiento de las relaciones entre los componentes de producción económica, los ámbitos políticos, las diferenciaciones con base en la raza y la legitimación de un saber por encima de otros en las repúblicas latinoamericanas nacidas en el siglo XIX, que permitió y todavía permite, la perpetuación de las relaciones de poder de tipo colonial ${ }^{8}$. En relación con los contenidos la pregunta es, entonces, ¿cómo se prescribe su trabajo en los programas de estudios de Historia y de Ética y Valores y qué relación tienen con la justicia social?

Ambos programas de estudio del BG incluyen colonialismo, pero no colonialidad. Historia lo hace a partir de la omisión y de estrategias adaptativas de la tradición nacionalista. Ética y Valores, por 
su parte, lo hace desde una posición científica con estrategias afirmativas y un dejo de historia crítica. En el caso de historia, sea Universal o de México, lo que predomina es una narración curricular teleológica y con intensa carga positivista, sobre todo en lo referente al pasado mexicano, donde los alumnos identifican "los sistemas y reglas o principios medulares que subyacen a una serie de fenómenos" históricos, como la caída de México Tenochtitlan (BG 2013c: 26). Estas leyes del acontecer histórico facilitan la prescripción de una narración histórica curricular que establece "un orden natural de las cosas", al explicar la conformación del Estado mexicano y la base mestiza de su identidad como condición necesaria.

Historia de México I, donde aparecen explícitamente los contenidos de la conquista de México y del periodo colonial, establece dos grandes consecuencias de este periodo colonial: la evangelización y el mestizaje. El mestizaje es un discurso teleológico que niega la pluralidad existente en Mesoamérica, simplificándola en un legado histórico y cultural homogéneo de los pueblos que habitaron México (BG 2013c: 24) y su aporte a la cultura nacional. Posteriormente, el programa de estudio establece una narración eurocéntrica a partir del descubrimiento de América y la versión europea de la conquista de México, para iniciar la etapa colonial con el estudio de las repercusiones de la conquista, de las cuales solo se menciona una: el mestizaje. Esta reubicación de la mirada histórica, de lo mesoamericano a lo español, permite construir un otro interno en México, una extranjería nacional, compuesta por los pueblos indios que se estudian como folclor. Quizá el ejemplo más claro de esto es cuando se aclara que "el descubrimiento de América es un hecho visto desde Occidente, y narrado por los vencedores. Sería conveniente contar con un texto de historia desde la perspectiva indígena, como La visión de los vencidos" (BG 2013c: 27). La oración, además de la perpetuación de lo indígena como otro comete dos errores básicos, pues confunde la interpretación con el hecho histórico y sugiere un libro de la conquista de México para estudiar el descubrimiento de América. En el caso de Historia de México II, que es la historia del Estado mexicano independiente, los indígenas simplemente desaparecen hasta 1994, con el levantamiento neozapatista (BG 2013d). La omisión es casi total, no únicamente de las condiciones de marginación, pobreza y exclusión de estas comunidades, sino de cualquier pluralidad cultural, religiosa o étnica mexicana. El problema no está en hablar de la diferencia, pues México está compuesto por múltiples otros, sino solo de otro, estableciendo una relación binaria donde lo indígena existe en cuanto contribuye a la cultura mestiza. Esto es una perpetuación de las oposiciones binarias que reproducen la desigualdad simbólica.

Al mirar hacia el exterior, la asignatura de Historia Universal Contemporánea es eurocéntrica. La presencia del colonialismo, por la temporalidad que abarca el programa, es poca. Los países colonizados, es decir, la mayor parte del mundo, se incluyen en la narración histórica curricular en cuanto explican la historia de las grandes potencias, en un nueva reproducción de los opuestos binarios. Pero también abren resquicios para trabajar la colonialidad si el profesor lo pretendiera. Por ejemplo se pide a los alumnos dibujar un mapa de África bajo el dominio imperial, para después "establecer la relación del mapa con una noticia actual sobre la pobreza imperante en África como consecuencia del imperialismo" (BG 2013e: 12-13). Aunque sugiere una relación directa pasado-presente, sin matices históricos intermedios, se puede afirmar que la colonialidad es trabajada en cuanto se señala la permanencia de las condiciones de miseria de las naciones como consecuencia de invasiones pasadas. También el resto del mundo emerge en las guerras de liberación nacional como India, Argelia, Irán, Nicaragua, entre otros, pero resaltando no el pensamiento o las causas políticas, sino "señalando los principales líderes políticos y sociales en estos procesos" (BG 2013e: 23). Lo anterior produce una interpretación ambivalente. Hay rastros de historia crítica al reconocer los movimientos de liberación nacional, pero esta huella se da dentro de la estructura de la historia nacionalista, elitista y memorística del código disciplinar de la historia.

Las asignaturas de Ética y Valores, comprendidas como las herederas del espacio curricular que ocupaba la formación cívica, muestran mucho más variaciones históricas que los programas de historia. El cambio de civismo a enseñanza de valores, es decir, del derecho a la filosofía, es positivo, a pesar de que este cambio omita aspectos importantes de la justicia social. La colonialidad es un ejemplo claro. El programa trabaja el tema en el bloque 4 de Ética y Valores I, dedicado a la identidad nacional ante los fenómenos asociados a la globalización. Ahí se parte, a diferencia del programa de Historia, de que México es una sociedad multicultural, por 
lo que hay que estudiar entre varios contenidos "los fenómenos históricos de dominio cultural, dominación hegemónica y colonización y las repercusiones en las conductas ocasionadas en la actualidad en la población mexicana" (BG 2013a: 30). Esta prescripción puede ser ubicada dentro de una tradición crítica, así como temas relacionados a los derechos humanos en Ética y Valores II, lo que nos lleva a pensar que promueven una estrategia transformadora. Sin embargo, no todo el programa es así, pues se imbrica con una idea de ciudadano liberal estrechamente atada a los conceptos de competencias que lo enmarcan en una estrategia afirmativa.

La enseñanza de la historia en el BG mexicano se ubica en el eje nacionalista, con pequeñas trazos de una educación científica basada en las competencias. La exclusión de la enseñanza crítica y la omisión deliberada de cualquier intento por romper con los opuestos binarios que dan sustento ideológico a la desigualdad, muestran que esta asignatura no ha sido concebida con contenidos potencialmente útiles para las estrategias transformativas, sino solo para las adaptativas. No así la formación ética y valoral, donde se rechaza la tradición nacionalista, para impartir un perfil científico con elementos críticos. Esto tiene que ver, entre otros aspectos, con los autores de los programas y la cultura disciplinar a la que pertenecen. El programa de estudio está hecho por los propios profesores del BG y supervisado por las autoridades, por lo que es posible pensar que son ellos mismos los responsables de la reproducción, dentro de esta escuela, de la desigualdad simbólica producida por la interpretación histórica, así como los responsables de los contenidos críticos de la formación ética. Esto implica que la perpetuación de la violencia simbólica en el currículo no necesariamente es un acto de imposición exterior, sino que el resultado es consecuencia más de la propia historia escolar que de la RIEMS. Lo anterior muestra, una vez más, que la función del maestro se ubica en la intersección entre la reproducción simbólica de la desigualdad y la lucha por la emancipación, y que es el docente dentro del aula quien escoge qué camino elegir.

\section{A modo de conclusión: Historia y ciudadanía en el bachillerato}

Entender el currículo en su dimensión histórica y política nos permite comprender cómo se imbrican diferentes posiciones teóricas en su interior. En el caso de la enseñanza de la historia y la formación ciudadana en el BG mexicano demuestra cómo cada asignatura, históricamente unidas en los programas de estudio, han tomado actualmente caminos distantes. Por un lado, la asignatura de historia mantiene, con una fidelidad alarmante, el código disciplinar. Da prioridad a la identidad nacional, reproduce la condición de subalternidad de los pueblos indígenas y las minorías, construye una narración teleológica $\mathrm{y}$ omite cualquier contenido crítico. Es un programa con enormes potencialidades adaptativas y, por tanto, contradictorias con el proyecto de competencias impuesto por la RIEMS, cuya lógica es afirmativa. No sucede así con la asignatura de ética y valores, donde los contenidos relacionados con la colionalidad promueven una reflexión acerca de las relaciones de poder en el pasado y en el presente, lo que da indicios de la permanencia de elementos críticos dentro del programa. En este tema, su estrategia es potencialmente transformativa, aunque al armonizarla con las competencias, la estrategia afirmativa también lucha por ocupar un lugar en el currículo.

La historicidad de ambas asignaturas nos muestra la imbricación de tradiciones históricas entre la enseñanza de la historia, por un lado, y la formación ética, ciudadana y moral, por otro. De esta manera, en un solo currículo podemos encontrar una historia nacionalista con estrategias afirmativas, interrelacionada con visiones científicas de corte adaptativo $\mathrm{y}$, en algunos aspectos, incluso posiciones críticas contra las formas de dominación en el presente. Sin embargo, la imbricación no logra armonizarse, pues las tradiciones de cada asignatura detertminan su proceder. En otras palabras, la propuesta de enseñanza de la historia reproduce el código disciplinar de la historia en la escuela, por lo que se explica por el propio pasado de la asignatura, no por la RIEMS. La imbricación se da en diversos temas, como el de la colonialidad. No hay posibilidad de congeniar la lógica excluyente $-y$ racista- de la homogeneidad mestiza con la idea de una nación pluricultural. Asimismo, no hay posibilidad de establecer una relación armónica entre una ciudadanía exclusivamente basada en la emoción y la raza, con una propuesta de un sujeto racional que actúa civilizadamente en los asuntos de la vida pública. Finalmente, al ser el contenido histórico una historia del Estado mexicano, y no de los múltiples Méxicos, se promueven estrategias adaptativas hacia una cultura preexistente, sin posibilidad de transformación. 
Es evidente que un programa de estudio tan amplio, y con tantos actores en juego, enfrenta un sinnúmero de dificultades para diseñarse con coherencia. Sin embargo, la diferencia entre formación ética y enseñanza de la historia en el BG sobrepasa, por mucho, simples errores técnicos. Para solucionar esto, considero fundamental recuperar el eje crítico de la enseñanza de la historia, sobre todo para incluir contenidos problemáticos del pasado y del presente, donde no se omitan los procesos de violencia, imposición y exclusión de las sociedad actuales y pasadas. Finalmente, urge definir con claridad qué tipo de ciudadano se quiere formar con la asignatura de historia, para no producir un texto curricular que afirme enseñar lo que no promueve y oculte la violencia que prescribe. Así, podríamos vislumbrar la posibilidad de que los contenidos de historia del Bachillerato General, en vez de reproducir las condiciones simbólicas de desigualdad social, promuevan la justicia social como parte sustancial de sus contenidos.

\section{Referencias Citadas}

Andreotti, V., y Souza, L. M.

2012 Postcolonial perspectives on global citizenship education. Routledge, Nueva York.

Argüello, D.

2017 Genealogía del pensamiento crítico en la enseñanza de la historia del nivel medio superior en la Ciudad de México, desde 1970 hasta la actualidad. Tesis doctoral en proceso. Mecanoescrito.

Bachillerato General

2013a Ética y Valores I. Subsecretaría de Educación Media Superior, México.

Bachillerato General

2013b Ética y Valores II. Subsecretaría de Educación Media Superior, México.

Bachillerato General

2013c Historia de México I. Subsecretaría de Educación Media Superior, México.

Bachillerato General

2013d Historia de México II. Subsecretaría de Educación Media Superior, México.

Bachillerato General

2013e Historia Universal Contemporánea. Subsecretaría de Educación Media Superior, México.

Barba, J.F.

2007 Las (Im)posibilidades de la Educación Ciudadana en México. Revista Electrónica Iberoamericana sobre Calidad, Eficacia y Cambio en Educación, 5: 51-69. http://www. rinace.net/arts/vol5num4/art3.pdf. Consultado 13/04/2016.

Barton, K. y Levstik, L.

2004 Teaching history for the common good: Lawrence Erlbaum Associates, Nueva Jersey.

Boisvert, J.

2004 La formación del pensamiento crítico: Teoría y práctica. Fondo de Cultura Económica, México.

Conde, S., Gutiérrez Espíndola, J. L. Chávez Romo, C.

2015 Cartilla ciudadana. Fondo de Cultura EconómicaPueblo Hacia Arriba Fundación, México.

Cuesta, R.

1998 Clío en las aulas. La enseñanza de la historia en España entre reformas, ilusiones y rutinas, Akal, Madrid.

DeLeon, A., y Ross, E. W.

2010 Critical theories, radical pedagogies, and social education: New perspectives for social studies education. Sense Publishers, Rotterdam.
Díaz Barriga, F., García, J. A.

2008 La comprensión de la noción de tiempo histórico en estudiantes mexicanos de Primaria y Bachillerato. Cultura y educación: revista de teoría, investigación y práctica 20 : 143-160.

Díaz Barriga Arceo, F.

1998 Una aportación a la didáctica de la historia en bachillerato. Perfiles educativos, 82: 115-132.

Díaz Barriga, Á.

2015 Currículum: entre utopía y realidad. Buenos Aires ; Madrid: Amorrortu.

Epstein, T.

2009 Interpreting national history: Race, identity, and pedagogy in classrooms and communities. Routledge, Nueva York.

Fraser, N.

2006 La justicia social en la era de la política de la identidad: redistribución, reconocimiento y participación. En ¿Redistribución o reconocimiento?: un debate políticofilosófico, Fraser, N. y Honneth, A., pp. 17-88. Morata, Madrid,

García Garduño, J.

2014 Estudio introductorio. Pinar, W. La teoría del curriculum. Madrid: Narcea, 11-60.

Lévesque, $\mathrm{S}$.

2008 Thinking historically: Educating students for the twenty-first century. University of Toronto Press, Toronto.

Meneses, E.

1998 Tendencias educativas oficiales en México 1821-1911. UIA, México, vol. 1.

Miranda, F.

2013 México, sin héroes y en la orfandad por festejos patrios: INEHRM, Milenio, México, 7 de agosto. Disponible en http://www.patriciagaleana.net/Mexico-sin-heroes.html, Consultado: 22/03/2016

O’Gorman, E.

1986 La superviviencia política novohispana. Monarquía y república. UIA, México.

Osborne, K.

2006 To the Past': Why We Need to Teach and Study History. En To the Past: History Education, Public Memory, and Citizenship in Canada, editado por Ruth W. Sandwell, 103-131. University of Toronto Press, Toronto. 
Pérez Rocha, M.

2011 Las humanidades y la barbarie de la RIEMS. La Jornada. México, 16 de junio. 03/04/2016 http://www.jornada.unam. Plá, S. $\mathrm{mx} / 2011 / 06 / 16 /$ opinion/020a2pol 03/04/2016

2016 Currículo, historia y justicia social. Un estudio comparativo en América Latina, Revista colombiana de educación. Ramírez, R.

1948 La enseñanza de la historia en México. Instituto Panamericano de geografía e historia, México.

Ross, E.

2001 The social studies curriculum: Purposes, problems, and possibilities. State University of New York Press, Albany.

Sánchez Quintanar, A.

2004 Reencuentro con la historia. Teoría y praxis de su enseñanza en México. Paideia-UNAM, México

Seixas, P., Morton, T., Colyer, J., y Fornazzari, S.

2013 The big six: Historical thinking concepts. Nelson Education, Toronto.

\section{SEP}

2008a Acuerdo Secretarial Número 442 por el que se establece el Sistema Nacional de Bachillerato en un marco de diversidad. DOF, México, 26 de septiembre
SEP

2008b ACUERDO número 444 por el que se establecen las competencias que constituyen el marco curricular común del Sistema Nacional de Bachillerato. DOF, México, 21 de octubre

Vázquez, J.

1970 Nacionalismo y educación. El Colegio de México, México.

Villa Lever, L.

2010 La Educación Media Superior: su construcción social desde el México independiente hasta nuestros días. En Arnaut, A. Y Giorguli, S. Los grandes problemas de México, El Colegio de México, México: 271-311.

Walsh, C.

2009 Interculturalidad, Estado, sociedad: Luchas (de) coloniales de nuestra época. Universidad Andina Simón Bolívar, Quito.

Zorrilla Alcalá, Juan Fidel.

2010 El bachillerato mexicano un sistema académico precario: causas y consecuencias. México, D.F.: Universidad Nacional Autónoma de México.

\section{Notas}

1 Para Raymundo Cuesta el código disciplinar es "una tradición social que se configura históricamente y que se compone de un conjunto de ideas, valores, suposiciones y rutinas, que legitiman la función educativa atribuida a la Historia" (Cuesta, 1998: 8). Dentro de él se alberga el "valor educativo, los contenidos de enseñanza y los arquetipos de práctica docente" (Cuesta, 1998: 8-9), que la cultura dominante considera valiosos y legítimos.

2 Una síntesis del proceso de reconceptualización curricular, puede verse en García Garduño (2014)

3 Las posibles relaciones entre los contenidos históricos y la formación valoral, ética y ciudadana son muchas. Incluso, las competencias genéricas del Marco Curricular Común (MMC) para la Educación Media Superior (EMS) las considera. Sin embargo, ni los programas de historia ni los de Ética y Valores las desarrollan a cabalidad. Por ejemplo, en Ética y Valores I solo se trabaja el tema de la colonización y la dominación cultural, mientras que en Ética y valores II solo se historiza a la bioética.

4 Esta propuesta retoma en su núcleo lo mencionado por Ken Osborne (2006) para el caso canadiense, pero a la vez se distancia de ella, mucho, sobre todo en que para este autor una etapa supera a otra: lo crítico supera al nacionalismo y lo científico a lo crítico.

5 Cabe destacar en este periodo la fundación del Colegio de Ciencias y Humanidades $(\mathrm{CCH})$ de la Universidad
Nacional Autónoma de México (UNAM). El enfoque de este centro puede enmarcarse en una estrategia transformativa, pues los principios políticos y críticos que lo fundaron tuvieron ese propósito explícito. Asimismo, en los aspectos pedagógicos como la interdisciplinariedad, la relación horizontal docente-alumno, la notoria carga curricular de las materias de las humanidades, en especial a la mirada histórica social y una interrelación muestra el saber hacer, el saber ser y en especial el aprender a aprender son muestra de ello.

6 Villa Lever divide esta etapa en dos, separando la reforma actual como un periodo particular. Otra narración de la historia del bachillerato, desde el concepto de anomia durkheimiano, puede verse en Zorrilla (2010).

7 Un caso a contracorriente se dio en la Ciudad de México, con la fundación del Instituto de Educación Media Superior, que creó planteles para la atención a los sectores más vulnerables de la ciudad. Su enfoque permite una atención personalizada a los estudiantes, tiene una pronunciada carga política heredera de la educación popular y los contenidos de humanidades giran en torno de la Historia.

8 Este resumen amerita la pluralidad teórica de la corriente decolonial. Existen muchos autores que han trabajado en profundidad el tema, como Walter Mignolo, Aníbal Quijano, Enrique Dussel y en el ámbito educativo Catherine Walsh. 
